\title{
Chronic $Q$ fever: An ongoing challenge in diagnosis and management
}

\author{
Ira Das MD FRCPath ${ }^{1}$, Nicola Guest Msc ${ }^{1}$, Richard Steeds MA MD FRCP FESC ${ }^{2}$, Peter Hewins MRCP PhD 3
}

\begin{abstract}
I Das, N Guest, R Steeds, P Hewins. Chronic Q fever: An ongoing challenge in diagnosis and management. Can J Infect Dis Med Microbiol 2014;25(1):35-37.

Chronic $Q$ fever is a potentially fatal disease. The current difficulty in the diagnosis of this condition is discussed in the present article. A 51-year-old woman with a history of aortic valve replacement presented with complaints of feeling generally unwell, pyrexia and occasional unproductive cough over a period of several weeks. Phase 1 immunoglobulin $\mathrm{G}$ titre to Coxiella burnetii was initially detected at a low level (1:320, detected using immunofluorescence) and was not considered to be significant according to the modified Duke criteria. Later in the course of her illness, the patient's antibody titre rose to a high level (1:1280). The issues regarding current laboratory diagnosis and management of $Q$ fever are discussed. Chronic $Q$ fever can be associated with an inadequate serological response. Close follow-up of cases is essential. The recommended serological criteria for the diagnosis of $Q$ fever endocarditis needs to be revisited.
\end{abstract}

Key Words: Echocardiogram; Endocarditis; Modified Duke criteria; Q fever serology

\begin{abstract}
○ fever is a zoonosis that occurs worldwide and is caused by Coxiella burnetii, an obligate intracellular Gram-negative bacterium (1). It is mainly known as an occupationally acquired infection in individuals who have had contact with domestic animals (2).

$Q$ fever has a high variability in clinical manifestation, although most cases are either asymptomatic $(60 \%)$ or mildly symptomatic with spontaneous recovery (2). Acute disease may range from flu-like syndrome to severe pneumonia and hepatitis (2), and may lead to serious complications, including death, especially in patients with meningoencephalitis or myocarditis and, more frequently, in cases with chronic infection associated with endocarditis (2). Up to $5 \%$ of patients with acute $Q$ fever develop endocarditis (3). Patients at risk for chronic $Q$ fever include individuals with cardiac valve pathology, aneurysms or vascular grafts and, to a lesser extent, immunocompromised hosts and pregnant women (2,3). Endocarditis is the most severe and potentially fatal form of chronic $Q$ fever. This is the most common cause of blood culture-negative endocarditis and comprises $3 \%$ to $5 \%$ of all endocarditis cases (4). We present a case of $Q$ fever endocarditis and highlight the difficulty with regard to diagnosis and management.
\end{abstract}

\section{CASE PRESENTATION}

A 51-year-old woman, who was hemodialysis dependent because of chronic renal failure, was admitted University Hospitals Birmingham (Birmingham, United Kingdom) in February 2011 with a history of fever, occasional unproductive cough and feeling generally unwell for several weeks, and feeling more unwell with high fever during the week before the admission. She was last discharged from the hospital two weeks previously, having been admitted with a history of pyrexia

\section{La fièvre $Q$ chronique : un défi constant sur le plan du diagnostic et du traitement}

La fièvre Q chronique est une maladie au potentiel fatal. Le présent article traite de la difficulté à la diagnostiquer. Une femme de 51 ans ayant des antécédents de remplacement de la valvule aortique a consulté parce qu'elle ne se sentait généralement pas bien et présentait une pyrexie et une toux non productive occasionnelle depuis plusieurs semaine. Les médecins ont d'abord décelé un faible taux du titre d'immunoglobuline en phase 1 à Coxiella burnetii (1:320, décelé par immunofluorescence), lequel n'était pas considéré comme significatif selon les critères de Duke modifiés. Plus tard au cours de l'évolution de la maladie, les titres d'anticorps de la patiente ont atteint un taux élevé (1:1280). Les auteurs explorent les enjeux relatifs au diagnostic en laboratoire et au traitement de la fièvre $Q$. La fièvre $Q$ chronique peut s'associer à une réponse sérologique inadéquate. Il est essentiel d'assurer un suivi étroit des cas. Il faudrait revoir les critères sérologiques recommandés pour diagnostiquer une endocardite à fièvre $\mathrm{Q}$.

${ }^{1}$ Department of Clinical Microbiology $\mathcal{E}$ Infection Control; ${ }^{2}$ Department of Cardiology; ${ }^{3}$ Department of Nephrology, University Hospitals Birmingham NHS Foundation Trust, Birmingham, United Kingdom

Correspondence: Dr Ira Das, Department of Clinical Microbiology $\mathcal{B}$ Infection Control, University Hospitals Birmingham NHS Foundation Trust, Birmingham B15 2WB, United Kingdom. Telephone 44-0-121-371-6172, fax 44-0-121-371-6540, e-mail ira.das@uhb.nhs.uk 
TABLE 1

$Q$ fever serology (immunofluorescence)

\begin{tabular}{lccccccccc}
\hline & December 3, & December 20, & March 2, & March 14, & May 16, & August 15, & October 17, & March 5, & July 25, \\
& $\mathbf{2 0 1 0}$ & $\mathbf{2 0 1 0}$ & $\mathbf{2 0 1 1}$ & $\mathbf{2 0 1 1}$ & $\mathbf{2 0 1 1}$ & $\mathbf{2 0 1 1}$ & $\mathbf{2 0 1 1}$ & $\mathbf{2 0 1 2}$ & $\mathbf{2 0 1 2}$ \\
\hline Phase 1 lgG & $1: 640$ & ND & $1: 320$ & $1: 1280$ & $1: 640$ & $1: 2560$ & $1: 320$ & ND & ND \\
Phase 1 lgA & ND & ND & ND & ND & ND & ND & ND & ND \\
Phase 2 lgG & $1: 2560$ & $1: 2560$ & $1: 1280$ & $1: 5120$ & $1: 2560$ & $1: 1280$ & $1: 1280$ & $1: 640$ & ND \\
Phase 2 lgM & ND & ND & ND & ND & ND & ND & ND & ND & ND \\
\hline
\end{tabular}

Ig Immunoglobulin; ND Not detected

following day and daptomycin was commenced. Gentamicin and rifampicin were continued. Eleven sets of blood cultures, including two drawn before the initiation of antibiotic therapy, remained negative.

At two weeks of admission, Q fever serology (indirect immunofluorescence) was reported to be positive in a low titre (Table 1). A repeat sample was advised and the current titre of antibodies was considered to be indicative of previous $Q$ fever infection; therefore, specific therapy against $Q$ fever was not commenced. She continued to be pyrexial, although her CRP level declined to $78 \mathrm{mg} / \mathrm{L}$ at three weeks of admission and she generally felt better. Noninfectious causes of endocarditis (marantic endocarditis) were considered. Antinuclear antibodies and antineutrophil cytoplasmic antibodies were not detected; C3 and C4 complement levels and serum immunoglobulin (Ig) G, IgA and IgM levels were tested and noted to be within the normal range, and her IgE level was below normal.

A computed tomography scan of the thorax, abdomen and pelvis on the 22 nd day of admission revealed mediastinal lymphadenopathy, cardiomegaly, a small right pleural effusion and ground-glass changes throughout the lungs (reported to be most likely due to fluid overload), a possible small infarct in her spleen and no evidence of neoplasia. A diagnosis of tuberculosis was considered. At six weeks of admission, because of continuing pyrexia, current antibiotics comprising daptomycin, gentamicin and rifampicin were considered to be inappropriate and were withdrawn after a period of 14 days. A combination of doxycycline $100 \mathrm{mg}$ twice daily and ciprofloxacin $500 \mathrm{mg}$ twice daily orally was commenced.

A stored sample of serum obtained before the current episode was sent to the reference laboratory for analysis, as well as additional serum samples for $Q$ fever serology, which subsequently showed a fourfold rise of phase I IgG titres in the latest sample (Table 1).

She remained intermittently pyrexial with a progressive decrease in CRP level at four weeks of admission. Malaria, Brucella, Bartonella and Toxoplasma serology and serum cryptococcal antigen tests were unremarkable or negative. Atypical pneumonia serology revealed a low titre of antibodies (1:32) against influenza A and B; antibodies to Mycoplasma pneumoniae, Chlamydia psittaci, respiratory syncytial virus and adenovirus were negative. Brucella and Toxoplasma serology were reported to be negative. She remained cardiovascularly stable, gradually became apyrexial with a significant overall improvement and normalization of CRP level. She was discharged home after five weeks of admission with advice to continue the abovementioned medications against $Q$ fever.

On outpatient review six weeks postdischarge, she was afebrile without any signs of heart failure, but was experiencing increasing symptoms of breathlessness. Her CRP level on this visit was noted to be within the normal range. She subsequently reported intolerance (nausea and vomiting ) to the antibiotics (doxycycline and ciprofloxacin) at 10 weeks postdischarge. Her medications were subsequently changed to a combination of doxycycline $100 \mathrm{mg}$ twice daily and hydroxychloroquine $200 \mathrm{mg}$ once daily orally. However, she was noted to have experienced significant weight loss by 12 weeks postdischarge, with complaints of nausea and loss of appetite although she reported that she had been compliant with the medications. She was readmitted four months post discharge. She was noted to be pyrexial (temperature $>38^{\circ} \mathrm{C}$ ) and her CRP level had risen to $297 \mathrm{mg} / \mathrm{L}$. Antibiotic therapy was again changed to a combination of ciprofloxacin $250 \mathrm{mg}$ twice daily and rifampicin $450 \mathrm{mg}$ twice daily orally. A repeat transesophageal echocardiogram on this admission revealed no abnormality. A repeat computed tomography scan of the thorax was unremarkable and resolution of the previously reported lymphadenopathy was noted. A repeat $Q$ fever serology on this admission showed a higher titre of phase I antibody (Table 1). She was subsequently noted to have a subacromial bursitis of her right shoulder and lymphadenopathy of the cervical and axillary regions. The possibility of a coexistent infective process, such as tuberculosis, was again considered. However, she experienced gradual improvement in her general condition, accompanied by reduction of her CRP level and occasional pyrexial periods. She was transferred to an infectious disease unit for a second opinion, where she continued on ciprofloxacin and rifampicin and was subsequently discharged home after two weeks with advice to continue the current medications. A transthoracic echocardiogram subsequently showed no paravalvular aortic regurgitation, normal left ventricular size and function. She was last followed up in the clinic on September 3, 2012, when she was noted to be generally well, apyrexial and her CRP level was within the normal range.

Histological examination of the cervical lymph node revealed reactive changes including sinus histiocytosis without any evidence of malignancy. Special staining, including Ziehl-Nelsen, Grocott, periodic acid-Schiff and Gram staining of the lymph node sections, were unremarkable. The lymph node tissue, fluid from the right shoulder bursa and biopsy of the right shoulder tissue were negative on culture including Mycobacteria. Shoulder tissue and the bursa fluid were reported negative for $\mathrm{C}$ burnetii by $16 \mathrm{~S}$ polymerase chain reaction (PCR), routine bacteria and Mycobacteria. Histology of the lymph node indicated reactive changes. The histological sections of the explanted aortic valve from 2000 were re-examined and noted to have chronic inflammatory changes; however, 16S PCR could not be performed because the tissue sample was not viable. The most recent $Q$ fever serology (one year after the initial presentation) revealed a negative phase I IgG titre (Table 1).

\section{DISCUSSION}

$Q$ fever endocarditis is the primary identifiable etiological agent of blood culture-negative endocarditis (4). Up to $5 \%$ of acute Q fever cases develop chronic infection after several months to years after an acute infections $(2,5)$. Infective endocarditis is the major manifestation of chronic Q fever, accounting for $60 \%$ to $80 \%$ of all chronic $Q$ fever cases $(2,3,6)$. Abnormal native or prosthetic valves are most commonly affected (1).

Clinical diagnosis of chronic Q fever, particularly endocarditis, remains difficult and is often delayed because the symptoms are nonspecific; the common criteria for the diagnosis of endocarditis, such as fever and positive echocardiographic findings, are frequently absent and routine blood cultures are negative (6).

The major histological features of endocarditis are minimal, absent or nonspecific, resembling a degenerative process (7). Cardiac vegetations, when present, are small and are associated with mononuclear cell infiltrations, extensive fibrosis and, frequently, calcification. The diagnosis is confirmed using serology. A delay in the diagnosis of chronic $Q$ fever is associated with a worse prognosis and higher mortality (8). When untreated, the disease is mainly associated with death and, even with appropriate treatment, is associated with a significant mortality of $10 \%$ at five years (6). Lymphadenopathy detected in our 
patient further complicated the clinical management by raising the possibility of tuberculosis/neoplasia. Lymphadenopathy and osteoarticular involvement are only rarely observed in $Q$ fever $(9,10)$.

The sensitivity of PCR testing of peripheral blood is variable and has not been evaluated prospectively. A history of exposure to animals is often absent, as in our case. The diagnosis of Coxiella endocarditis is, therefore, often complex. Currently, the diagnosis is mainly based on positive serological tests. The original Duke criteria, developed for the diagnosis of infective endocarditis, include vegetations and positive blood cultures as major criteria. However, vegetations are usually absent and conventional blood cultures are negative in $Q$ fever. The modified Duke criteria include a positive result on $\mathrm{Q}$ fever immunofluorescence assay of phase $1 \mathrm{IgG}$ antibody titre $\geq 1: 800$ in the major criteria for the diagnosis of endocarditis (11). The $\operatorname{IgG}$ antibody titre in our patient, when first tested at presentation, was below this level and was, therefore, interpreted to be of uncertain significance. Although the patient had risk factors for chronic $Q$ fever and a probable cardiac vegetation, her low titre of phase $1 \mathrm{IgG}$ antibody combined with a lack of prompt and sustained response to specific antimicrobial treatment against $Q$ fever led to difficulty in the management.

Discrepancies among the results of serological tests from different reference laboratories was reported by Healy et al (12) in a study involving the follow-up of 102 patients after a point source outbreak in Newport, United Kingdom, further contributing to the challenges in the diagnosis of chronic $Q$ fever.

In the largest cohort of $Q$ fever endocarditis published to date, Million et al (6) identified the association of prosthetic valve infection with higher mortality, frequent stroke, delayed serological cure, need for longer treatment course and higher risk of relapse. On the basis of these data, the optimum management includes a longer course of therapy (24 months) with doxycycline and hydroxychloroquine for prosthetic valve infections. Intolerance to the recommended drugs can be a problem, as illustrated in our case, which further complicates

\section{REFERENCES}

1. Marrie T, Raoult D. Coxiella burnetii (Q fever) In: Mandell GL, Bennett JE, Dolin R, eds. Mandell, Douglas and Bennett's Principles and Practice of Infectious Diseases, 7th edn. Philadelphia: Elsevier, 2010;2511-19.

2. Maurin M, Raoult D. Q fever. Clin Microbiol Rev 1999;12:518-53.

3. Brouqui P, Dupoint HT, Drancourt M, et al. Chronic Q fever:

Ninety two cases from France, including 27 cases without endocarditis. Arch Intern Med 1993;153:642-48.

4. Fournier P-E, Thuny F, Richet H, et al. Comprehensive diagnostic strategy for blood cuture-negarive endocarditis: A prospective study of 819 new cases. Clin Infect Dis 2010;51:131-40.

5. Landais C, Fenollar F, Thuny F, Raoult D. From acute Q fever to endocarditis: Serological follow-up strategy. Clin Infect Dis 2007;44:1337-40

6. Million M, Thuny F, Richet H, Raoult D. Long-term outcome of Q fever endocarditis: A 26-year personal survey. Lancet Infect Dis 2010;10:527-35.

7. Lepidi H, Houpikian P, Liang Z, Raoult D. Cardiac valves in patients with $Q$ fever endocarditis: Microbiological, molecular and histologic studies. J Infect Dis 2003;187:1097-106. the management. Although the patient reported compliance with the medications, her initial lack of response to treatment containing doxycycline may have resulted from inadequate absorption of the antibiotics due to frequent vomiting. The combination of rifampicin with a fluroquinolone has been shown to be effective in vitro and has been used in cases of endocarditis $(1,13)$. Our patient tolerated the above combination, with clinical and serological improvement.

In the absence of close follow-up with serological results for $\mathrm{C}$ burnetii, the diagnosis of chronic $\mathrm{Q}$ fever would have been missed because of initial low titre of phase I IgG antibody. We assume that deficient immunological status due to chronic renal failure and hemodialysis contributed to the initial lack of adequate antibody response and to the protracted course despite appropriate antibiotic therapy.

\section{CONCLUSION}

Chronic $Q$ fever endocarditis may be associated with an atypical presentation and have a protracted course in spite of antibiotic therapy. Serological response in chronic $Q$ fever may be associated with a low or fluctuating antibody titre. The possibility of chronic $Q$ fever should be considered as a cause of an unexplained fever and should not be dismissed in the presence of an inadequate or atypical serological response, especially in immunosuppressed patients. Careful clinical and serological follow-up is essential in high-risk patients to detect its evolution over time. More research is needed to improve laboratory diagnosis, including reconsideration of the recommended serological criteria for the diagnosis of $\mathrm{Q}$ fever endocarditis.

ACKNOWLEDGEMENTS: The authors thank the HPA Microbiology laboratory (Porton Down, Salisbury, Wiltshire, United Kingdom) for providing the Coxiella burnetii serology and molecular diagnostic service.

DISCLOSURES: The authors have no conflicts of interest to declare. Ethical approval was not required for this work. No funding was obtained for this work.

8. Raoult D, Etienne J, Massp P, et al. Q fever endocarditis in the south of France. J Infect Dis 1987;155;:570-3.

9. Foucault C, Lepidi H, Poujet-Abadie JF, et al. Q fever and lymphadenopathy: Report of four new cases and review. Euro J Clin Microbiol Infect Dis 2004;23:759-64.

10. Leonetti F, Raoult D, Dussol B, Brunet P, Berland Y. Chronic Q fever in hemodialysis patients. Nephron 1994;67:231-3.

11. Li JS, Sexton DJ, Mick N, et al. Proposed modifications to the Duke criteria for the diagnosis of infective endocarditis. Clin Infect Dis 2000;30:633-8.

12. Healy B, Llewelyn M, Westmoreland D, Lloyd G, Brown N. The value of follow-up after acute $Q$ fever infection. J Infect 2006;52:e109-12.

13. Yeaman MR, Roman MJ, Baca OG. Antibiotic susceptibilities of two Coxiella burnetii isolates implicated in distinct clinical syndromes. Antimicrob Agents Chemother 1989;33:1052-7. 


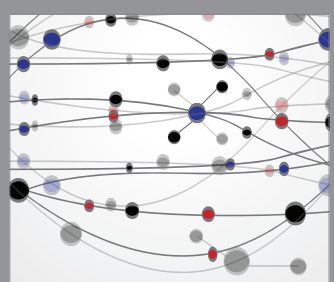

The Scientific World Journal
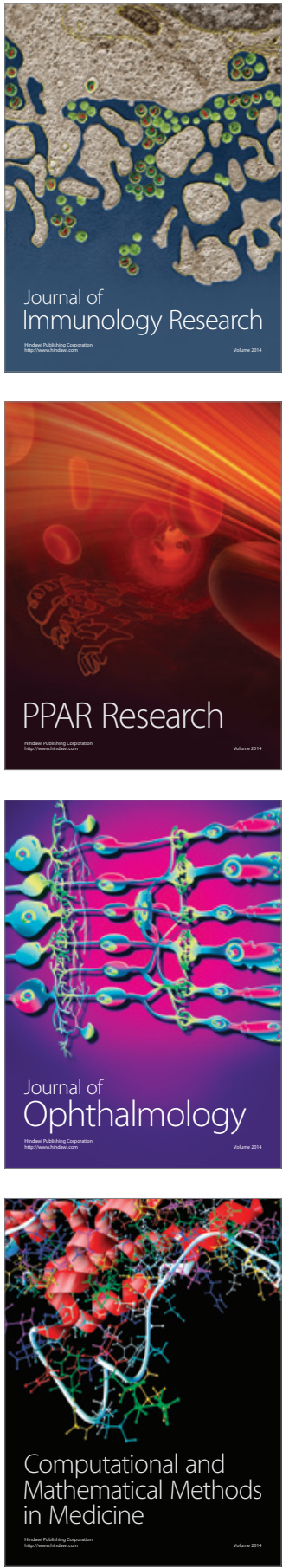

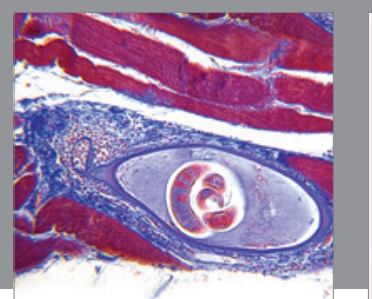

Gastroenterology Research and Practice

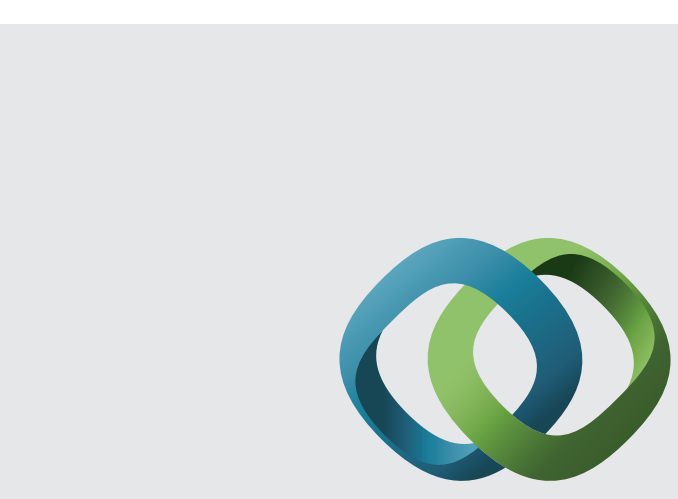

\section{Hindawi}

Submit your manuscripts at

http://www.hindawi.com
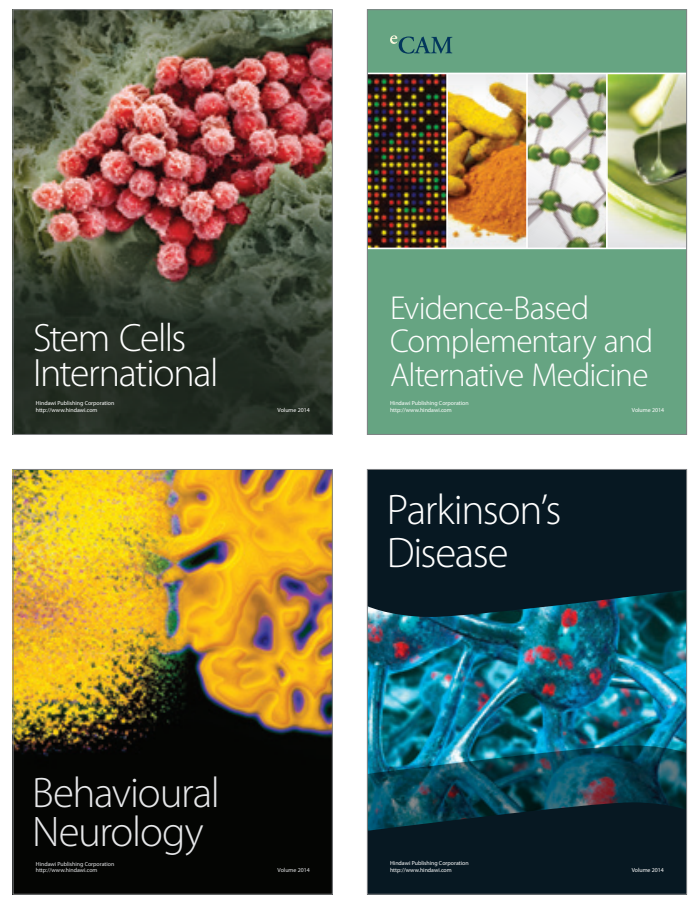
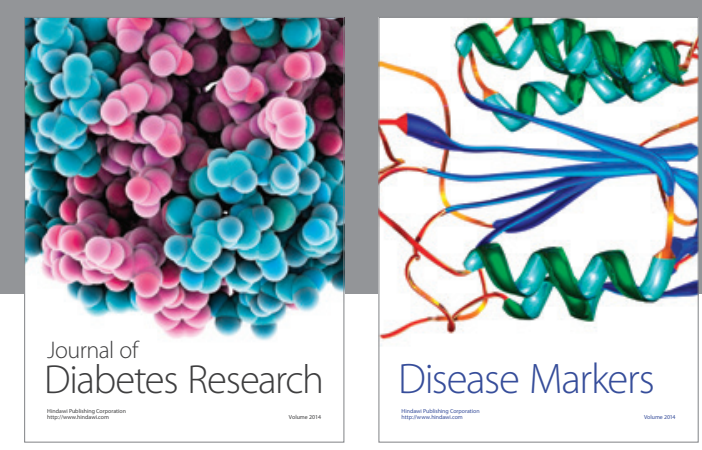

Disease Markers
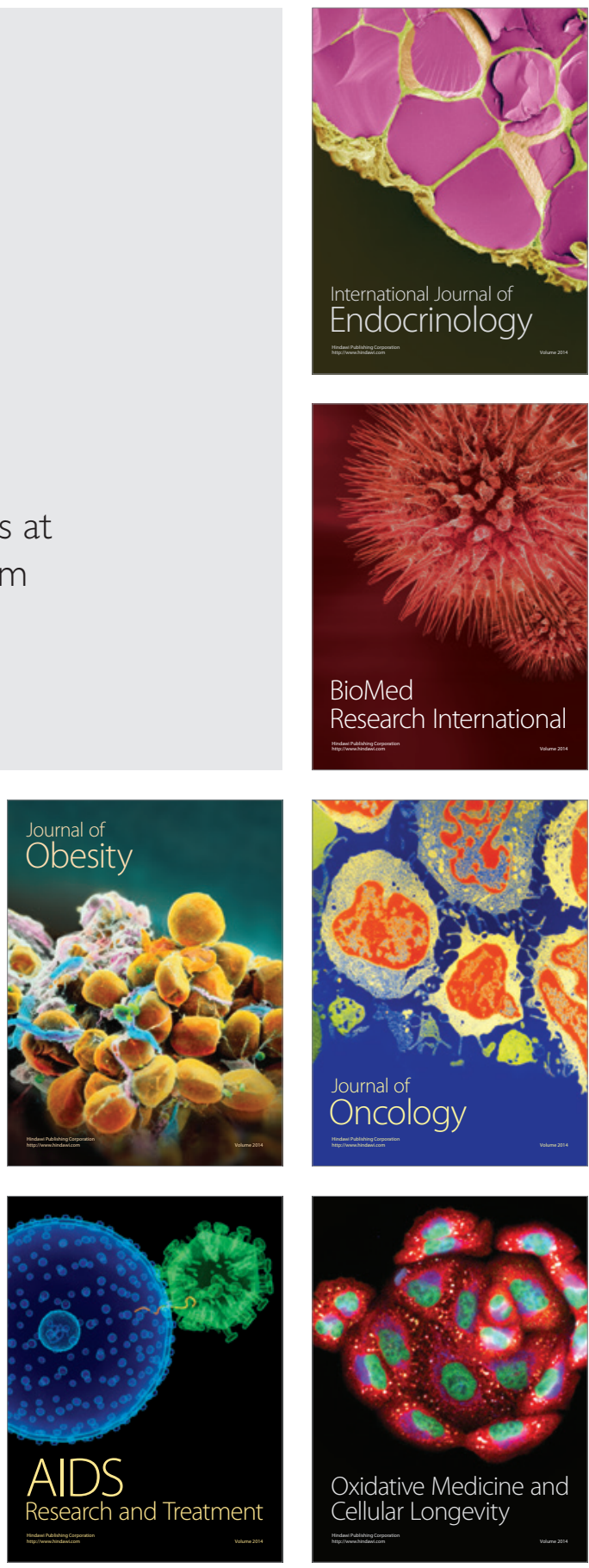